Published in final edited form as:

Angew Chem Int Ed Engl. 2017 July 17; 56(30): 8801-8804. doi:10.1002/anie.201702898.

\title{
A Hybrid Nanomaterial for the Controlled Generation of Free Radicals and Oxidative Destruction of Hypoxic Cancer Cells
}

\section{Prof. Song Shen,}

The Wallace H. Coulter Department of Biomedical Engineering, Georgia Institute of Technology and Emory University, Atlanta, GA 30332 (USA). College of Pharmaceutical Sciences, Jiangsu University, Zhenjiang, Jiangsu 212013 (China)

\section{Dr. Chunlei Zhu,}

The Wallace H. Coulter Department of Biomedical Engineering, Georgia Institute of Technology and Emory University, Atlanta, GA 30332 (USA)

\section{Dr. Da Huo,}

The Wallace H. Coulter Department of Biomedical Engineering, Georgia Institute of Technology and Emory University, Atlanta, GA 30332 (USA)

\section{Miaoxin Yang,}

School of Chemistry and Biochemistry, Georgia Institute of Technology, Atlanta, GA 30332 (USA)

Dr. Jiajia Xue, and

The Wallace H. Coulter Department of Biomedical Engineering, Georgia Institute of Technology and Emory University, Atlanta, GA 30332 (USA)

\section{Prof. Younan Xia}

The Wallace H. Coulter Department of Biomedical Engineering, Georgia Institute of Technology and Emory University, Atlanta, GA 30332 (USA). School of Chemistry and Biochemistry, Georgia Institute of Technology, Atlanta, GA 30332 (USA)

\section{Abstract}

\begin{abstract}
Anticancer modalities based on oxygen free radicals, including photodynamic therapy and radiotherapy, have emerged as promising treatments in the clinic. However, the hypoxic environment in tumor tissue prevents the formation of oxygen free radicals. Here we introduce a novel strategy that employs oxygen-independent free radicals generated from a polymerization initiator for eradicating cancer cells. The initiator is mixed with a phase-change material and loaded into the cavities of gold nanocages. Upon irradiation by a near-infrared laser, the phasechange material is melted due to the photothermal effect of gold nanocages, leading to the release and decomposition of the loaded initiator to generate free radicals. The free radicals produced in this way are highly effective in inducing apoptosis in hypoxic cancer cells.
\end{abstract}

\section{Oxygen-independent free radicals}

Correspondence to: Younan Xia.

Supporting information and the ORCID identification number(s) for the author(s) of this article can be found under http:// dx.doi.org/XXX/XXX 
Gold nanocages filled with a phase-change material (PCM) are used as a carrier for AIPH to achieve controlled generation of free radicals, which is oxygen-independent and can be used for the therapy of hypoxic cancer.

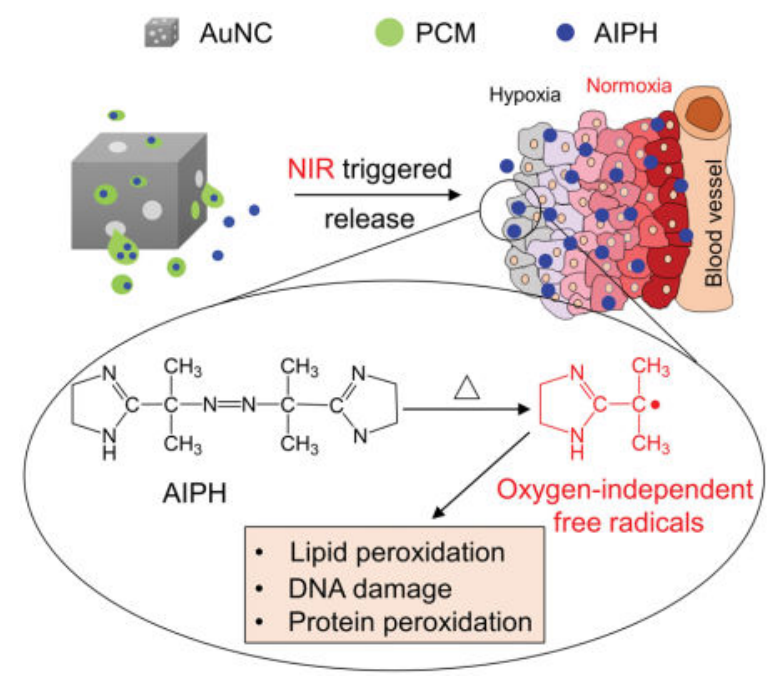

\section{Keywords}

photothermal; free radicals; Au nanocages; AIPH; phase-change material (PCM)

Reactive oxygen species (ROS), including peroxide, superoxide, hydroxyl radical, and singlet oxygen, can kill cells by causing oxidative damage to lipids, proteins, and DNA. Cancer treatment based on ROS has been widely adopted for clinical applications in the form of radiotherapy, ${ }^{[1]}$ photodynamic therapy (PDT), ${ }^{[2]}$ sonodynamic therapy (SDT),${ }^{[3]}$ and chemotherapy. ${ }^{[4]}$ For all these anticancer modalities, the therapeutic effect is critically dependent on the local concentration of oxygen. Most tumors tend to develop a hypoxic region, ${ }^{[5]}$ especially in the area far away $(>70 \mu \mathrm{m})$ from the nutritive blood vessels. ${ }^{[6]}$ Prior studies have established that cells in such a hypoxic region are highly resistant to chemo- ${ }^{[7]}$ and radiotherapy. ${ }^{[8]}$

One possible solution to the aforementioned issue is to deliver a precursor compound into the tumor for in situ generation of radicals upon activation. Here we report the demonstration of 2,2' -azobis[2-(2-imidazolin-2-yl) propane] dihydrochloride (AIPH) as a promising candidate for such an application. AIPH is a water-soluble azo compound that decomposes rapidly under thermal or irradiation stimulation to generate alkyl radicals. ${ }^{[9]}$ These radicals are toxic toward cells by directly oxidizing cell components or reacting with oxygen to generate secondary cytotoxic free radicals, including both alkoxyl and peroxyl radicals. ${ }^{[10]}$ Even under hypoxic condition, the radicals can still be generated to increase intracellular lipid hydroperoxides and thus induce apoptosis. ${ }^{[11]}$ However, AIPH is chemically unstable due to its susceptibility to decomposition in vivo, which inevitably reduces its availability at the tumor site and increase the side effects. In this study, we demonstrate the use of Au nanocages (AuNCs) filled with AIPH and a phase-change material (PCM) to achieve controlled generation of free radicals for cancer therapy. 
Figure 1a shows a schematic procedure for AIPH/PCM loading and controlled generation of free radicals. When irradiated with a near-infrared (NIR) laser, the heat generated through the photothermal effect of AuNCs melts the PCM, causing the encapsulated AIPH to be released and decomposed for the rapid generation of free radicals. The AuNCs were synthesized using the galvanic replacement reaction between $\mathrm{Ag}$ nanocubes and $\mathrm{HAuCl}_{4}$ according to a published method. ${ }^{[12]}$ The average edge length of the resultant AuNCs was $60 \pm 12 \mathrm{~nm}$, together with a wall thickness of $c a .7 \mathrm{~nm}$. Figure $1 \mathrm{~b}$ shows a transmission electron microscopy (TEM) image of the AuNCs, revealing small pores of 10-30 nm in size on the walls. If needed, the size of pores in the walls of AuNCs could be varied by adjusting the amount of $\mathrm{HAuCl}_{4}$ added, in an effort to optimize the loading and release of AIPH/ PCM. ${ }^{[12]}$ We then loaded a mixture of AIPH and PCM into the cavities of AuNCs by slightly modifying a previously reported protocol. ${ }^{[13]}$ Briefly, the AuNCs were dispersed in methanol, followed by the dissolution of PCM (lauric acid, m. p. $=44-46{ }^{\circ} \mathrm{C}$ ) and AIPH. The loaded AuNCs (denoted as Au-PCM-AIPH) were retrieved by centrifugation, followed by gentle washing with methanol. Compared with the pristine sample, the loaded AuNCs exhibited a noticeable difference in contrast when imaged under TEM (Figure 1, b and c). The differential scanning calorimetry (DSC) curves shown in Figure 1d confirm that PCM was successfully loaded into the cavities of AuNCs. Figure 1e shows the data of a thermogravimetric analysis (TGA) of the loaded AuNCs in the range of $50-600{ }^{\circ} \mathrm{C}$, giving a loading amount of $13.9 \%$ (by wt.) for the AIPH/PCM mixture. We further determined the loading capacity of AIPH by UV-vis spectroscopy. The weight percentage of AIPH in AuPCM-AIPH was about $7.9 \%$, which implies a weigh percentage of $6.0 \%$ for the PCM.

We investigated the release of AIPH from the loaded AuNCs with or without NIR laser irradiation. As shown in Figure 2a, the release of AIPH could be controlled by varying the power density and/or the duration of laser irradiation. A higher laser power density corresponds to a higher temperature and thus a faster release of AIPH from the interiors of AuNCs. After laser irradiation for $20 \mathrm{~min}$, the cumulative release percentages could reach 22.1 and $34.7 \%$ at power densities of 0.2 and $0.4 \mathrm{~W} \cdot \mathrm{cm}^{-2}$, corresponding to equilibrium temperatures of 54 and $68{ }^{\circ} \mathrm{C}$, respectively. In contrast, only $6.3 \%$ of AIPH was released without laser irradiation due to the limited diffusion of molecules through the solid PCM. ${ }^{[13,14]}$ We then characterized the release and decomposition of AIPH by measuring the concentration of 2,2' -azino-bis(3-ethylbenzothiazoline-6-sulfonic acid) free radicals $\left(\mathrm{ABTS}^{+}.\right)$, which were formed through a reaction between ABTS and the free radicals from AIPH. The generated ABTS ${ }^{+}$. exhibited a characteristic absorbance between 500-950 nm. As shown in Figure $2 \mathrm{~b}$, time-dependent generation of $\mathrm{ABTS}^{+}$. was observed when ABTS was incubated with Au-PCM-AIPH at 37 and $44^{\circ} \mathrm{C}$, respectively. The concentration of $\mathrm{ABTS}^{+}$. at $44{ }^{\circ} \mathrm{C}$ was significantly higher than that at $37^{\circ} \mathrm{C}$, suggesting the faster release and decomposition of AIPH at a higher temperature.

The radicals from the initiator can react with various cell components, leading to DNA damage, lipid peroxidation, and protein degradation. ${ }^{[15]}$ In the present work, we used red blood cells (RBCs) as a model to characterize the generation of AIPH radicals. As one of the end products of lipid peroxidation, methane dicarboxylic aldehyde (MDA) was measured as a marker of cell damage induced by Au-PCM-AIPH. RBCs incubated with Au-PCM-AIPH showed noticeable lipid peroxidation and increase of MDA level (Figure 2c). The higher 
concentration of Au-PCM-AIPH used, the more MDA generated. Meanwhile, NIR laser irradiation imposed a significant impact on MDA generation. Upon NIR laser irradiation for $30 \mathrm{~min}$, the amount of MDA was increased by 1.2, 1.5, and 1.7 fold at a concentration of $0.25,1$, and $2 \mathrm{mg} \cdot \mathrm{mL}^{-1}$, respectively, while MDA was only increased by $1.1,1.3$, and 1.4 fold in the group without laser irradiation. The generated AIPH radicals were able to disrupt the membrane, making it more permeable and thus inducing hemolysis. It should be pointed out that NIR irradiation alone in the absence of Au-PCM-AIPH did not induce hemolysis. We also quantitatively analyzed the hemolysis by incubating RBCs with Au-PCM-AIPH at 37 and $44{ }^{\circ} \mathrm{C}$, respectively. After incubation at $37^{\circ} \mathrm{C}$ for $3 \mathrm{~h}$, the influence of Au-PCMAIPH on RBCs was negligible and no obvious hemolysis was observed (Figures $2 \mathrm{~d}$ and S1). When incubated at $44{ }^{\circ} \mathrm{C}$ for $3 \mathrm{~h}$, the sample differed noticeably from the other groups, exhibiting deep red supernatant as an indicator of hemolysis. The absorbance at $410 \mathrm{~nm}$ was about five times as strong as what was observed in the absence of Au-PCM-AIPH (Figure 2d).

The cellular uptake of Au-PCM nanoparticles in A549 human lung cancer cells was studied by doping the PCM with rhodamine 6G to obtain Au-PCM-RG. As shown in Figure S2, the cellular uptake of Au-PCM-RG was time-dependent. After incubation for $4 \mathrm{~h}$, Au-PCM-RG mainly located in lysosomes. Since tumor hypoxia limits the generation of oxygen free radicals, it is important to demonstrate that AIPH free radicals could still be generated in a hypoxic environment. Using $2^{\prime}, 7^{\prime}$-dichlorodihydrofluorescein diacetate (DCFHDA) as an indicator, we examined the amount of AIPH-based free radicals generated in both hypoxia and normoxia. As shown in Figures 3a and S3, A549 cells treated with Au-PCM-AIPH in normoxia exhibited bright fluorescence after NIR irradiation. Fluorescence with a similar intensity was also observed in cells treated in hypoxia, indicating that the generation of free radicals was independent of oxygen level. In the control group with Au-PCM-AIPH alone, only weak fluorescence could be detected under similar conditions. This result indicates that AIPH could not be released from AuNCs in the absence of NIR laser irradiation. For the group treated with free AIPH, the fluorescence was also very weak, which was probably due to the limited uptake of free AIPH by cells. Even with NIR irradiation, we did not observe any significant enhancement in fluorescence intensity. We then investigated the apoptosis of A549 cells, as induced by the radicals from Au-PCM-AIPH, using membrane blebbing as an indicator, which usually occurs during cell apoptosis and necrosis. The blebs were stained red with a lipophilic membrane dye Dil. As shown in Figure 3b, blebs appeared in both normoxia- and hypoxia-treated cells after 4 and $8 \mathrm{~h}$. Meanwhile, cell shrinkage was observed compared with the control group. These results suggest that the AIPH radicals are able to effectively induce cell apoptosis under both normoxic and hypoxic conditions.

The therapeutic effect of Au-PCM-AIPH was further evaluated using morphological changes to the actin filaments (F-actin). For the group without laser irradiation, the F-actin (stained with Phalloidin-Alexa Fluor 555, red) was well-organized and highly elongated (Figure 4a). Upon irradiation for $10 \mathrm{~min}$, the F-actin became retracted and condensed for both normoxia and hypoxia groups. Significant damage to the cytoskeleton was observed in the group with laser irradiation for $30 \mathrm{~min}$, which was similar to the cells treated with free AIPH for $12 \mathrm{~h}$ (Figure S4). We then quantified the laser-triggered cytotoxicity of Au-PCM-AIPH nanoparticles against A549 cells using 3-(4,5-dimethyl-2-thiazolyl)2,5-diphenyl-2H- 
tetrazolium bromide (MTT) assay. When the concentration of Au-PCM-AIPH was increased, the cell viability gradually decreased (Figure 4b). Upon NIR irradiation, AuPCM-AIPH showed significantly enhanced capability in killing cells (with $60.5 \%$ of the cells being killed) compared with that without irradiation (only 35.5\% of the cells being killed), indicating the rapid generation of free radicals. Only minor cytotoxicity was detected in the group treated with empty AuNCs (Figure S5a). The irradiation of NIR did not cause additional cytotoxicity when the temperature was controlled below $45^{\circ} \mathrm{C}$. In the free AIPH group, concentration-dependent cytotoxicity was found and the NIR laser irradiation showed no further impact on cell viability (Figure S5b). It is worth noting that both lauric acid and poly(vinyl pyrrolidone) (PVP), a colloidal stabilizer used for the synthesis of AuNCs, have been widely used in drug delivery and can be considered as biocompatible materials. ${ }^{[16]}$

The cytotoxicity of Au-PCM-AIPH was also evaluated under hypoxic condition. As shown in Figure 4c, Au-PCM-AIPH exhibited comparable inhibition rate toward A549 cells in both hypoxic (61.1\%) and normoxic groups (62.3\%), indicating that the cytotoxicity of Au-PCMAIPH was independent of oxygen level. This result is consistent with a previous report where alkyl radicals aggravated cell apoptosis under low oxygen pressure. ${ }^{[11]}$ As a control, we studied the influence of oxygen on photodynamic therapy (PDT), an oxygen-dependent technique for killing cancer cells. After irradiation for $5 \mathrm{~min}\left(40 \mathrm{~mW} \cdot \mathrm{cm}^{-2}\right)$, protoporphyrin (PPIX) under normoxia showed a cell inhibition rate of $81 \%$ at a concentration of 20 $\mu \mathrm{g} \cdot \mathrm{mL}^{-1}$ (Figure $4 \mathrm{c}$ ). In contrast, a pronounced decrease in inhibition rate $(58 \%)$ was found under hypoxia. The PDT effect could also be observed by staining the dead cells with trypan blue (Figure S6). These results clearly demonstrate that PDT-induced cell death greatly relies on oxygen concentration while AIPH-triggered cell death is independent of oxygen.

In conclusion, we have demonstrated a hybrid nanosystem based on AuNCs, PCM, and a conventional polymerization initiator (AIPH) for the generation of free radicals in the absence of oxygen. This new platform offers a number of advantages, including enhanced stability, controlled release, and photo-triggered therapeutic effect. Different from radiotherapy and chemotherapy that are highly dependent on the oxygen level, the AIPHbased therapy can also destruct cancer cells in the absence of oxygen, holding promise for the treatment of hypoxic tumors. As a proof-of-concept study, the emphasis of this work was placed on the demonstration of an oxygen-independent strategy for the treatment of hypoxic tumors. The efficacy still needs to be improved by optimizing the components and conditions involved. On one hand, new initiators with sharp initiation temperatures should be developed to enable more swift and efficient generation of free radicals. On the other hand, the loading efficiency of the initiator should be improved by adjusting the loading protocols in an effort to reduce the amount of AuNCs administered. In addition, a multiple cancer killing mode that combines both photothermal therapy and the generation of free radicals can also be used to further augment the treatment efficacy.

\section{Supplementary Material}

Refer to Web version on PubMed Central for supplementary material. 


\section{Acknowledgments}

This work was supported in part by a grant from the NIH (R01 CA138527) and startup funds from Georgia Tech. As a visiting scholar, S.S. was also partially supported by a fellowship from the China Scholarship Council.

\section{References}

1. a) Zhang C, Zhao K, Bu W, Ni D, Liu Y, Feng J, Shi J. Angew Chem Int Ed. 2015; 54:17701774.Angew Chem. 2015; 127:1790-1794.b) Song G, Chen Y, Liang C, Yi X, Liu J, Sun X, Shen S, Yang K, Liu Z. Adv Mater. 2016; 28:7143-7148. [PubMed: 27275921]

2. a) Idris NM, Gnanasammandhan MK, Zhang J, Ho PC, Mahendran R, Zhang Y. Nat Med. 2012; 18:1580-1585. [PubMed: 22983397] b) Huang H, Yu B, Zhang P, Huang J, Chen Y, Gasser G, Ji L, Chao H. Angew Chem Int Ed. 2015; 54:14049-14052.Angew Chem. 2015; 127:14255-14258.

3. a) Li J, He Y, Sun W, Luo Y, Cai H, Pan Y, Shen M, Xia J, Shi X. Biomaterials. 2014; 35:36663677. [PubMed: 24462358] b) Shen S, Wu L, Liu J, Xie M, Shen H, Qi X, Yan Y, Ge Y, Jin Y. Int J Pharm. 2015; 486:380-388. [PubMed: 25841570]

4. a) Wong DY, Ong WW, Ang WH. Angew Chem Int Ed. 2015; 54:6483-6487.Angew Chem. 2015; 127:6583-6587.b) Ramanathan B, Jan KY, Chen CH, Hour TC, Yu HJ, Pu YS. Cancer Res. 2005; 65:8455-8460. [PubMed: 16166325]

5. Sullivan R, Graham CH. Cancer Metast Rev. 2007; 26:319-331.

6. Vaupel P, Hockel M, Mayer A. Antioxid Redox Sign. 2007; 9:1221-1235.

7. a) Samanta D, Gilkes DM, Chaturvedi P, Xiang L, Semenza GL. Proc Natl Acad Sci. 2014; 111:E5429-E5438. [PubMed: 25453096] b) Comerford KM, Wallace TJ, Karhausen J, Louis NA, Montalto MC, Colgan SP. Cancer Res. 2002; 62:3387-3394. [PubMed: 12067980]

8. Bertout JA, Patel SA, Simon MC. Nat Rev Cancer. 2008; 8:967-975. [PubMed: 18987634]

9. Yoshida Y, Itoh N, Saito Y, Hayakawa M, Niki E. Free Radic Res. 2004; 38:375-384. [PubMed: 15190934]

10. Krishna MC, Dewhirst MW, Friedman HS, Cook JA, Degraff W, Samuni A, Russo A, Mitchell JB. Int J Hyperthermia. 1994; 10:271-281. [PubMed: 8064185]

11. Chen Z, Saito Y, Yoshida Y, Niki E. Biosci Biotechnol, Biochem. 2008; 72:1491-1497. [PubMed: 18540111]

12. Skrabalak SE, Au L, Li X, Xia Y. Nat Protoc. 2007; 2:2182-2190. [PubMed: 17853874]

13. a) Moon GD, Choi SW, Cai X, Li W, Cho EC, Jeong U, Wang LV, Xia Y. J Am Chem Soc. 2011; 133:4762-4765. [PubMed: 21401092] b) Sun T, Wang Y, Wang Y, Xu J, Zhao X, Vangveravong S, Mach RH, Xia Y. Adv Healthcare Mater. 2014; 3:1283-1291.

14. Sun T, Zhang YS, Pang B, Hyun DC, Yang M, Xia Y. Angew Chem Int Ed. 2014; 53:1232012364.Angew Chem. 2014; 126:12520-12568.

15. a) Yoshida Y, Itoh N, Saito Y, Hayakawa M, Niki E. Free Radic Res. 2004; 38:375-384. [PubMed: 15190934] b) Saito Y, Nishio K, Yoshida Y, Niki E. Toxicology. 2005; 210:235-45. [PubMed: 15840437] c) Zhang H, Chen T, Jiang J, Wong Y, Yang F, Zheng W. J Agric Food Chem. 2011; 59:8683-8690. [PubMed: 21761878]

16. a) Higa OZ, Rogero SO, Machado LDB, Mathor MB, LugaÄ AB. Radiat Phys Chem. 1999; 55:705-707.b) Wissinga SA, Kayserb O, Müller RH. Adv Drug Deliv Rev. 2004; 56:1257-1272. [PubMed: 15109768] 
a
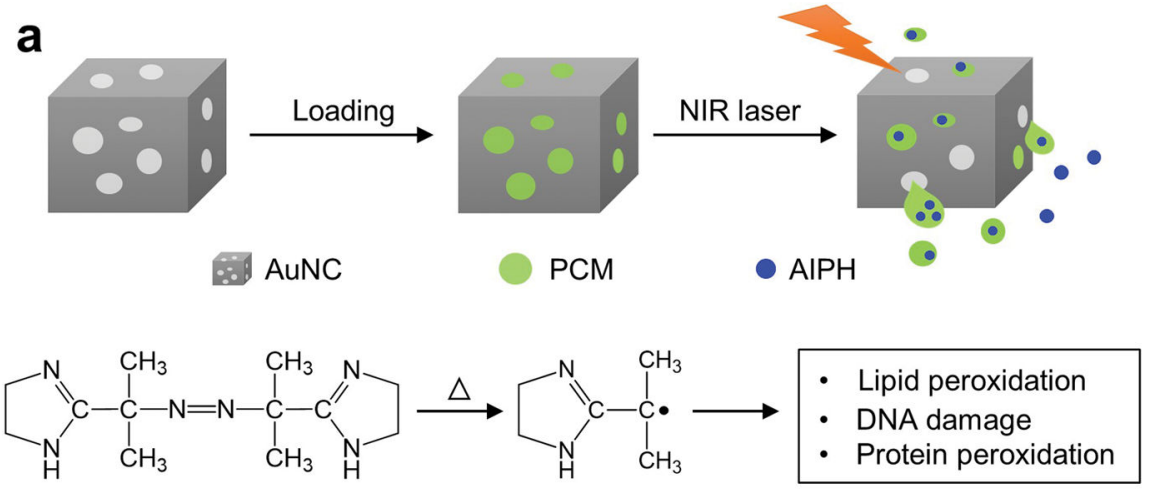

AIPH

Free radicals
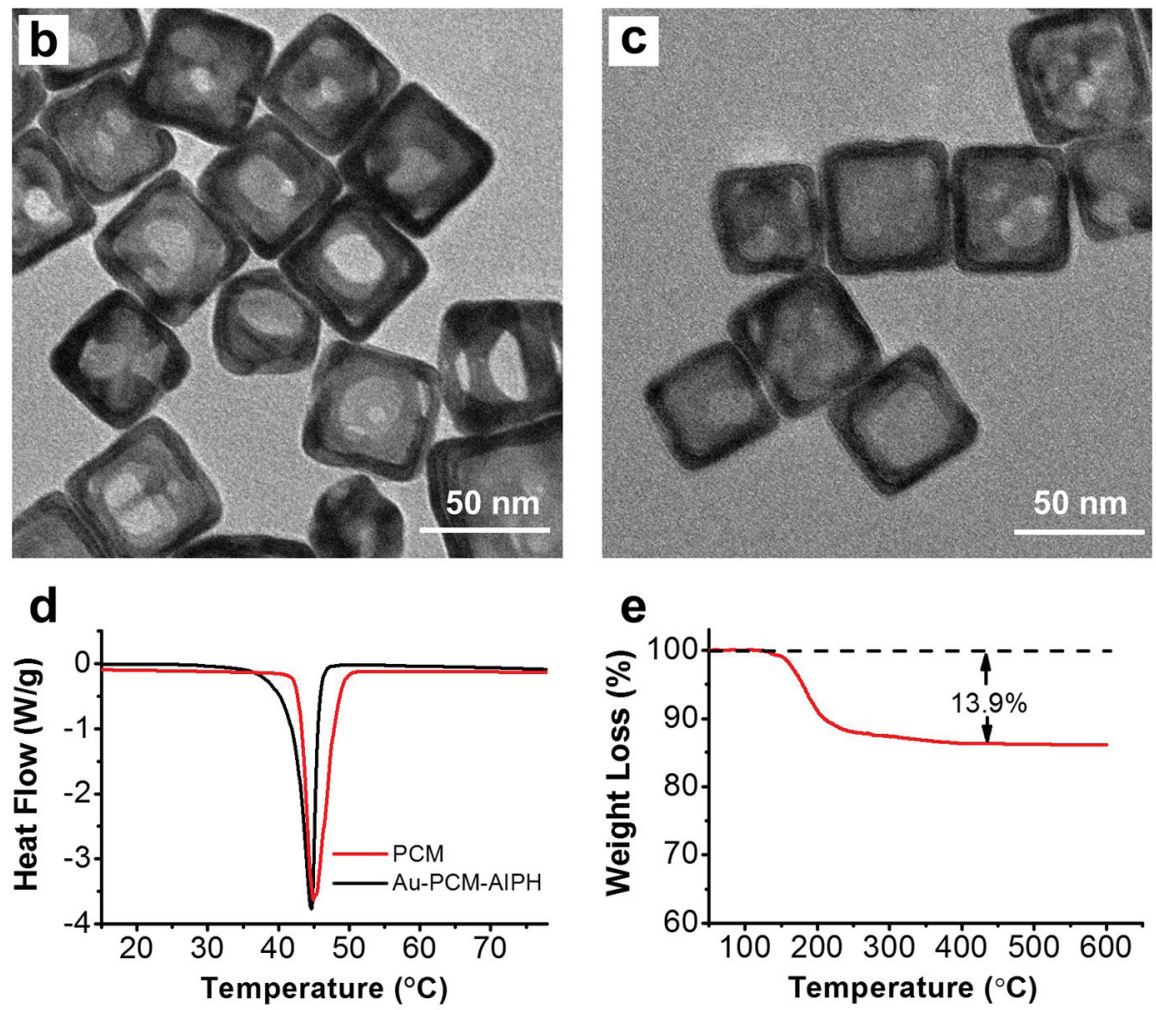

Figure 1.

a) Schematic illustration showing the controlled release and generation of free radicals upon irradiation by a NIR laser. b, c) TEM images of AuNCs (b) before and (c) after loading of the PCM-AIPH mixture. d) DSC curves recorded from the PCM and Au-PCM-AIPH samples, respectively. e) TGA curves obtained for the empty AuNCs (dashed) and PCMAIPH-loaded AuNCs (solid). 
a

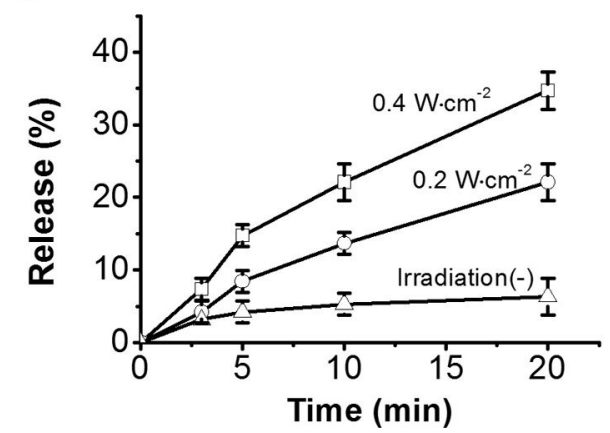

C

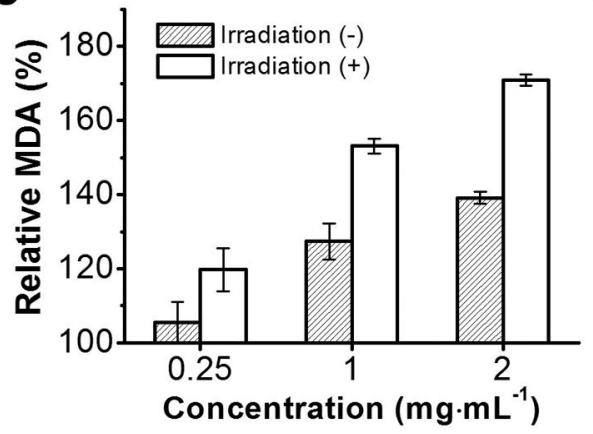

b

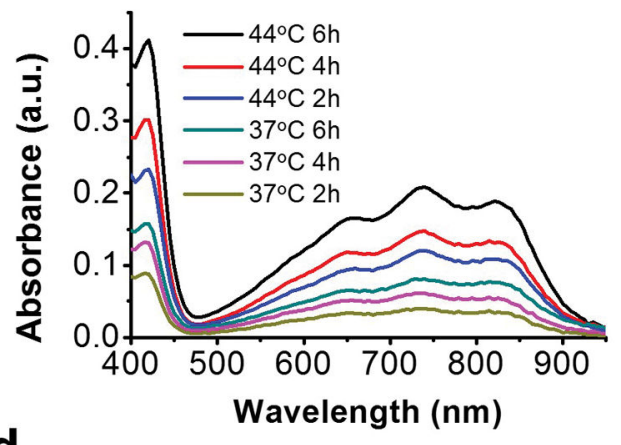

d

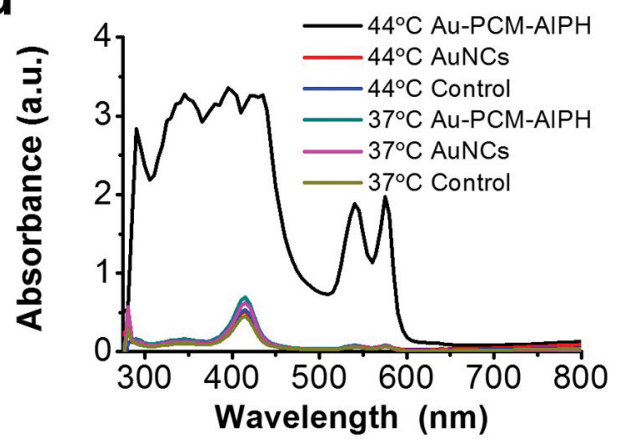

Figure 2.

a) Release profiles of AIPH from Au-PCM-AIPH under the irradiation of a NIR laser at different power densities. b) Generation of $\mathrm{ABTS}^{+}$. as induced by the free radicals released from Au-PCM-AIPH at different temperatures. c) Changes to MDA content in RBCs treated with different concentrations of Au-PCM-AIPH with or without NIR laser irradiation. d) UV-Vis absorption spectra of RBCs incubated with empty AuNCs $\left(1.5 \mathrm{mg} \cdot \mathrm{mL}^{-1}\right)$ and AuPCM-AIPH $\left(1.5 \mathrm{mg} \cdot \mathrm{mL}^{-1}\right)$ at 37 and $44{ }^{\circ} \mathrm{C}$, respectively, for $3 \mathrm{~h}$. 
a
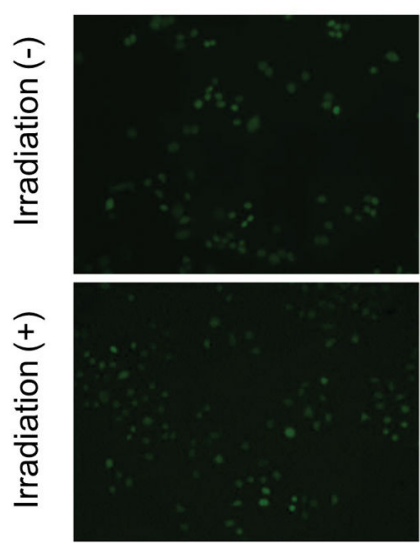

b
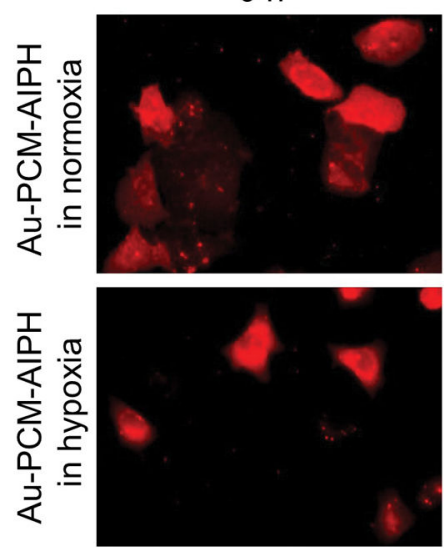

Au-PCM-AIPH in normoxia

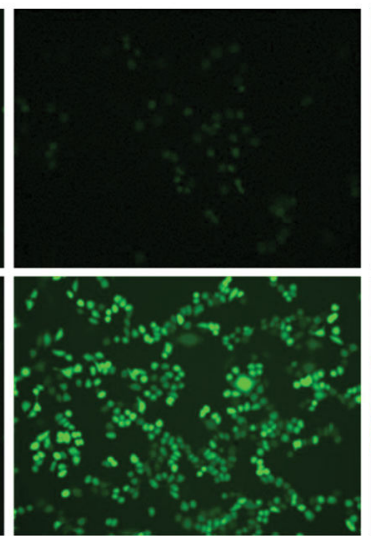

$4 \mathrm{~h}$
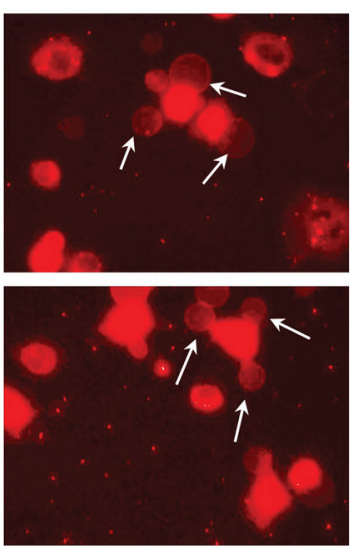

Au-PCM-AIPH in hypoxia

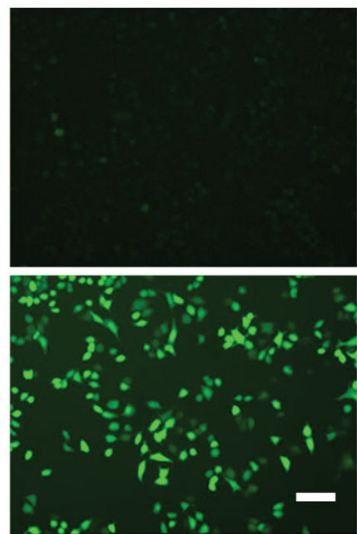

$8 \mathrm{~h}$
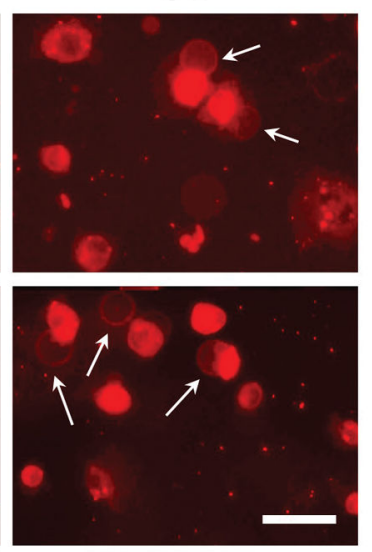

Figure 3.

a) Detection of AIPH-induced ROS in A549 cells by DCFHDA staining. The A549 cells were treated with AIPH $\left(20 \mu \mathrm{g} \cdot \mathrm{mL}^{-1}\right)$ or Au-PCM-AIPH (AIPH: $\left.20 \mu \mathrm{g} \cdot \mathrm{mL}^{-1}\right)$ in normoxic and hypoxic culture media, respectively, for $2 \mathrm{~h}$ and then irradiated by an $808-\mathrm{nm}$ laser for $30 \mathrm{~min}$. Scale bar $=50 \mu \mathrm{m}$. b) Morphology of A549 cells after incubation with Au-PCMAIPH (AIPH: $20 \mu \mathrm{g} \cdot \mathrm{mL}^{-1}$ ) for $2 \mathrm{~h}$ and irradiated with an 808-nm laser for $30 \mathrm{~min}$, and then incubated for another $4 \mathrm{~h}$ and $8 \mathrm{~h}$ in normoxic and hypoxic media, respectively. The cells were stained with a lipophilic membrane dye Dil to visualize the morphological change. Scale bar $=50 \mu \mathrm{m}$. 
a

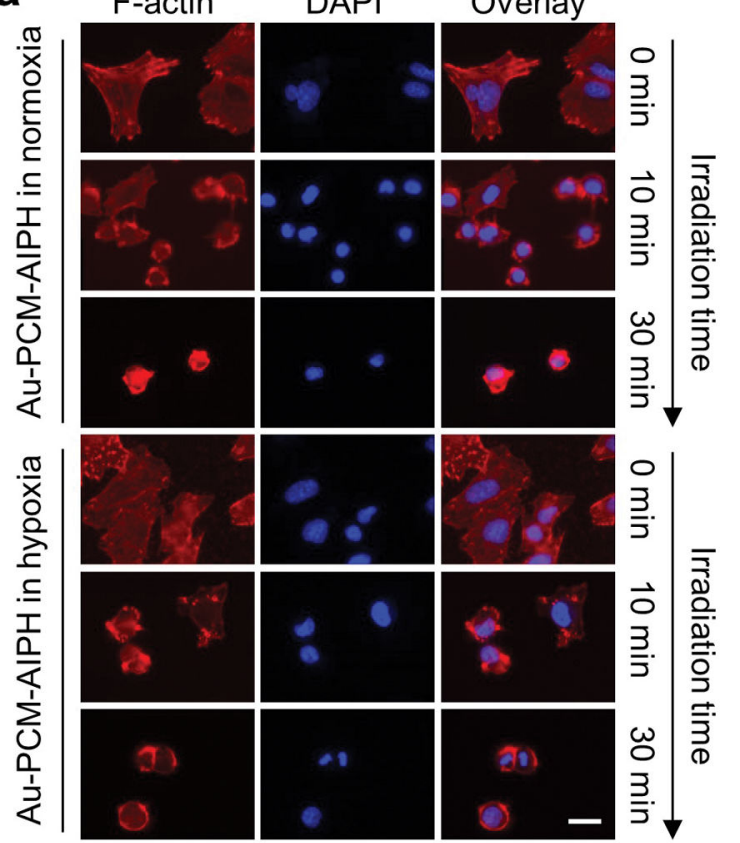

b

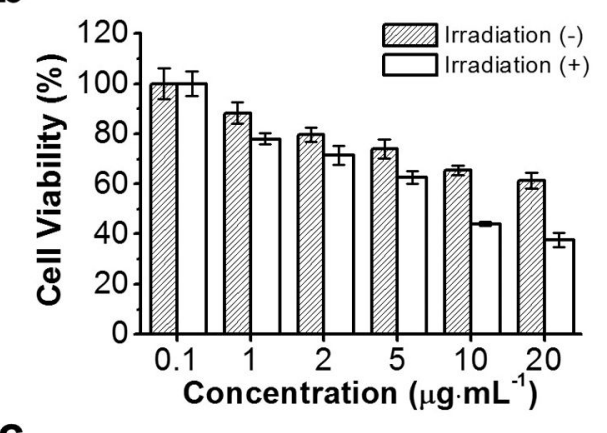

C

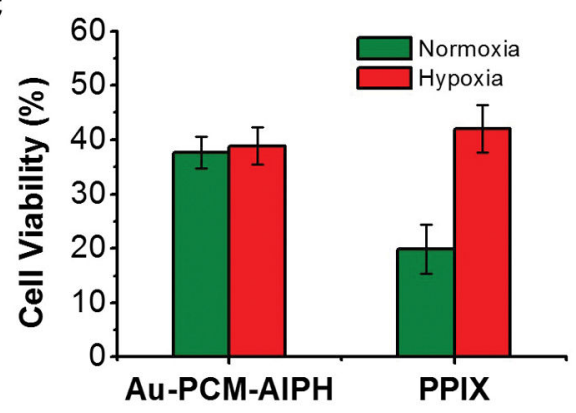

Figure 4.

a) F-actin morphology of A549 cells incubated with Au-PCM-AIPH in normoxic and hypoxic media under irradiation for different times. The cells were stained with PhalloidinAlexa Fluor 555 (a F-actin staining dye, red) and DAPI (a nuclear staining dye, blue). Scale bar $=25 \mu \mathrm{m}$. b) Cell viability after treatment with different concentrations of Au-PCMAIPH with or without NIR laser irradiation for $20 \mathrm{~min}$. Values represent mean \pm standard deviation $(n=6)$. c) Cell viability of A549 cells treated with Au-PCM-AIPH (AIPH: 20 $\left.\mu \mathrm{g} \cdot \mathrm{mL}^{-1}\right)$ or PPIX $\left(20 \mu \mathrm{g} \cdot \mathrm{mL}^{-1}\right)$ for $2 \mathrm{~h}$ and then irradiated with an $808-\mathrm{nm}$ or $405-\mathrm{nm}$ laser in normoxic and hypoxic media. The values were represented as mean \pm standard deviation $(\mathrm{n}=6)$ 